\title{
Accelerometer Based Structural Health Monitoring System on the Go: Developing Monitoring Systems with NI LabVIEW
}

\author{
https://doi.org/10.3991/ijoe.v15i07.10427
}

\author{
Salami Ifedapo Abdullahi $\left.{ }^{\bowtie}\right)$, Nurul Arfah Che Mustapha, \\ Mohamed Hadi Habaebi and Md. Rafiqul Islam \\ International Islamic University Malaysia (IIUM), Kuala Lumpur, Malaysia \\ Yakobud999@gmail.com
}

\begin{abstract}
Structural Health Monitoring (SHM) is a very crucial part of maintenance and management of buildings and structures. The use of SHM in recent years has been increasing due to the advancement in technology and the availability of nanodevices and nanosensors which can detect damaged part or crack in a structure. In this paper, PSpice simulation was carried out to show the response of the integrated electronic piezoelectric (IEPE) with a VPWL-source. Then, practical experiment was done using Arduino Mega with the ADXL335 accelerometer in a laboratory setup. LabVIEW software was used along with Arduino IDE software to make graphical visualization of accelerometer reading to be captured. Furthermore, a web service was deployed which enabled LabVIEW data transmission to a smartphone running Data Dashboard application for real-time monitoring anywhere. Therefore, making the system an ecosystem of Internet of Things enabling the user to access monitoring system while on the move. The result of the vibration test on the accelerometer showed that the accelerometer response to small changes in the $\mathrm{x}, \mathrm{y}$ and $\mathrm{z}$ axis of the accelerometer which can be used to detect micro-movements in a structure.
\end{abstract}

Keywords - Structural Health Monitoring (SHM), Accelerometer, ADXL335, LabVIEW, LabView Interface for Arduino (LIFA), Virtual Instruments (VI), Internet of Things (IoT)

\section{Introduction}

Structural Health Monitoring(SHM) are the use of sensors and devices in monitoring, analysing and maintaining building and bridges built with concrete and steel materials. SHM is the technique used to predict and detect damage for early warning as well as to ensure safety of structures [1].The other method is nondestructive testing(NDT) which are non-invasive means to determine the integrity of a 
material or structure and quantitatively measure some characteristics of structures [1]. Human activities and environmental effects are key factors that causes damages to reinforced concrete and steel structures such as cracks, delamination and corrosion.Overloading on top of building and structures are example of human activities that causes structural damage while corrosion on metallic structures is part of the example of environmental factors. There are many methods to build a system for SHM and it includes ultrasonic waves, electrical capacitance measurement, vibrations, electrical impedance, fiber Bragg wavelength shift, vision-based imagery and acoustic emission. Ultrasonic waves generate frequencies higher than $20 \mathrm{KHz}$ and are classified as either guided, surface or bulk waves [1]. Ultrasonic based SHM used an actuator to generate waves while the sensors measure the response of the structure through the waves which enables ultrasonic waves detects changes in propagation of waves through body of structures.

Capacitance based SHM consists of a capacitance sensor for detecting any changes in structure which is then measured by the changes in the capacitance [2]. MicroElectroMechanical Sensors(MEMS) accelerometer have low power outputs, are small in size and can measure frequency range of about $0-300 \mathrm{~Hz}$ [3]. SHM using vibrationbased sensors measures the vibration response of the structure and accelerometers are the most common type of sensors used to measure vibration [3-5]. Vibration based SHM detects changes in the stiffness, damping and through the mass of structures. [3] used an accelerometer, strain gauge and moisture sensor for an embedded system of SHM. The strain gauge gives electrical resistance based on amount of load or strain in the structure. Moisture sensor exploits the electrical resistance or dielectric constant of materials as they vary with moisture content. [4] presented state-of-the-art self-made MEMS accelerometer as a suitable tool for SHM of constructed facilities and systems. The assembled prototype of MEMS accelerometer has main components which include a logic unit, an accelerometer, an analog-to-digital converter(ADC), a micro SD memory card and a real time clock to make an agile system. To create an effective health monitoring of large structures, a very robust signal processing is needed for a very detailed data acquisition to detect, locate and predict damage and its severity in the structure [5]. After signal acquisition, signal processing is carried out and its used to extract features from the sensed data. [5] also discussed new methods of signal processing which are used to processed sensed data.

A successful design of SHM system needs to consider different aspects of the structure to be examined such as physical constraints, sensing capabilities, installation constraints, economics and human interfaces [6]. Therefore, task definitions indicate expectations of engineers from the SHM systems and the possible constraints that may exist during operation. Aesthetics is concerned with the use of sensors that causes minimal visual impact while calibration of sensors should automatic and serviceability of sensors should include sensors that can withstand severe loading. Maintenance and repair of SHM should be affordable and extra security should be incorporated in SHM to protect analysed data. Energy consumption of SHM should be renewable as well and [7] did an extensive study on the design of self-generated power capacitance measurement circuit for wireless health monitoring system. The energy harvester converts vibrations into electrical energy where hybrid approach of 
thermal energy harvester is used in a complement to obtain continuous power supply. Furthermore, [8] did extensive research on damage detection in real time using two materials embedded on a piezo-electric ceramic lead zirconate titanate(PZT). The composite material AI 6061-T6 and twill weave carbon fibre-reinforced epoxy are bonded together with the PZT to capture signal response with changes in crack depth [8].

Fiber Bragg grating is a periodic and permanent modification of the core refractive index value along the optical fibre axis [9]. FBG can be easily embedded into materials or composite materials to be part of SHM in providing damage detection or internal strain of structures. FBG works by inducing Bragg wavelengths shift or resonance when under temperature changes or strain. The techniques used to connect embedded fibers with structures includes bare optical fibers inside a loose tube with protection at edge of the composite material, edge connectors of FBGs and free space connection with an optical interrogator unit within a few meters away [9]. [10] studied the potential of camera enabled optical vision displacement sensor to demonstrate high accuracy of the vision sensor for full displacement measurement which can detect damage. Real-time capturing of target frame location is identified as template and re-located using template matching techniques. The displacement in pixels or subpixel is obtained which is then transformed into physical displacement in millimetres through a scaling factor [10]. An acoustic emission converts mechanical energy carried by elastic wave into an electrical energy [11]. The most widely used transducer in acoustic emissions is piezo-electric which makes only two types of acoustic emission available which are resonance and broadband sensors [11]. Parametric analysis and signal waveform analysis are two types of structural assessment approaches based on acoustic emission. Damage localization process and source discrimination are types of damage assessment carried out on reinforced concrete [11].

[12] extensively investigated acoustic emission waveforms of crack growth which occur even though conventional statistics- based analysis of acoustic emission have been carried out. The crack possesses the means for the acoustic emission in the material to propagate in the structure. [13] studied the sensitivity of active-sensing acousto-ultrasound-based SHM method to detect damage and identify the cause of sensitivity of the sensors. SHM involves four functional level referred to as technology classification levels [13]. The first level is for detection of the occurrence of an event. The second level is identification of geometric location of the event.The third level is for determination of the magnitude or severity of the event. The fourth level is the estimation of the remaining service life or strength. The technology needs to be advanced enough to effectively cater for the target action.

The motivation behind the investigation carried out in this paper is to extend previous researches involving SHM while enhancing the system to include a user interface to make SHM more interoperable. In this paper, a simulation to assess integrated electronic piezoelectric (IEPE) performance was carried out using OrCAD CapturePSpice simulation software which progressed into practical experimentation using an off-the-shelf accelerometer to assess its performance when it's being used in an SHM system. Furthermore, LabVIEW and NI Data Dashboard wereused as 
software enabler alongside Arduino IDE to evaluate the performance of the system and deploy monitoring system. The objective of the study is to investigate the micromovement in a structure using an accelerometer while being able to deploy a real-time monitoring system. Fig.1 showed a schematic representation of a generic active sensing SHM principle of operation on structures. The bridge and the building were created inside TinkerCAD 3D free online design suite. The figure depicts a structure health monitoring process which involves inspection by sensors, detection of faults or cracks and the alert or notification by the health management system.

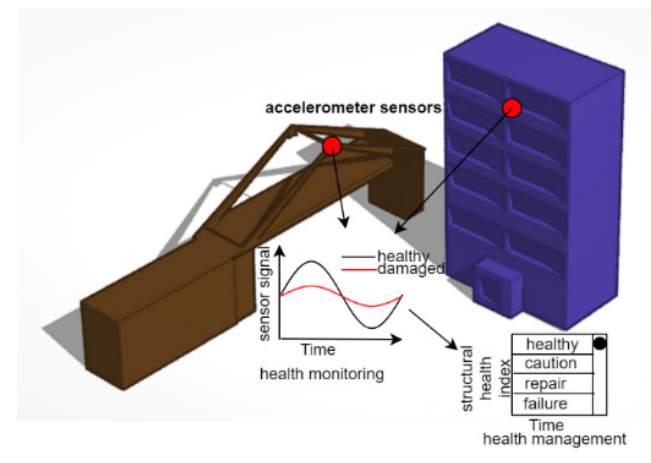

Fig. 1. Principle of operation of a generic active sensing SHM

\section{Methodology}

\subsection{OrCAD capture PSpice simulation}

Orcad 10.5 was the software utilised for the simulation of the IEPE circuit. The software utilized which is the Orcad Cadence is a good CAD software to display the connection of circuit diagram where visualization is needed before physical and actual construction. The orcad capture was used in the design process to make a schematic circuit of the IEPE interface circuit which consists the LT1636/LT,AD8631/AD and 2PA1576R/PLP. The IEPE transducer carries with it a voltage amplifier. The charge produce by IEPE transducer is too small. This charge needs to be pre-amplified so that it is not susceptible to noise, and it is condition to the circuit for better noise immunity. The IEPE interface can be recognized as a circuit to help in the analog to digital conversion before further analysis of the accelerometer voltage output. The IEPE interface circuit job is to synthesis the flow of circuit so that the signal from the accelerometer can be maintained at stable current.

The LT1636/LT is a difference amplifier, while the AD8631/AD is an operational amplifier and the 2PA1576R/PLP is a BJT Transistor. The other electrical components include the resistors and capacitors. The capacitors were to hold voltages while the resistors were to resist current flow. The LT1990 is to compare the data 
gained from sensor while the AD8628 in amplifier gain and the BJT is used to amplify the electrical signal from the sensor. The circuit was connected to a VPWLsource which can get data from a text file to input into the Orcad PSpice to get a graphed simulation of the voltage output. The VPWL-File source gets data from a text save file and then run it in OrCAD PSpice. The VPWL-FILE acts as the accelerometer sensor that gives data to the orCAD PSpice simulation. Fig. 2 shows the IEPE interface circuit constructed inside OrCAD and Fig. 3 shows the text file for the data storage acting as input to the VPWL-source. Fig.2 shows the circuit sketch which includes the connection of the virtual amplifiers and transistor along with resistors to showcase the circuit that amplifies the signal from an accelerometer. Fig.3 is an arbitrary data fed into the VPWL-source to enable a signal response in PSpice simulation.

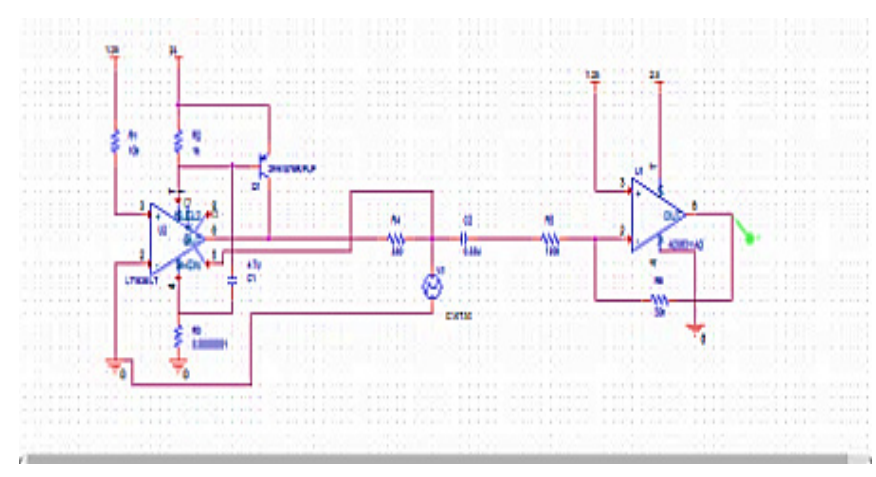

Fig. 2. IEPE interface circuit

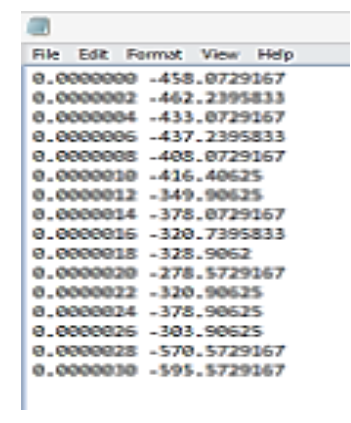

Fig. 3. VPWL-FILE source for data input

\subsection{Practical experimentation with accelerometer}

The accelerometer used in this experiment is ADXL335. The ADXL335 is a 3-axis analog-output accelerometer and its maximum capability is in the range of $\pm 3 \mathrm{~g}$ in 
which $1 \mathrm{G}$ is equal to $9.08 \mathrm{~m} / \mathrm{s}^{2}$. The accelerometer is placed on a mounted boardEVAL-ADXL335Z. Fig. 4 shows the schematic of EVAL-ADXL335Z board [14].Fig. 4 depicts EVAL-ADXL335Zwhich has pins consisting of ST, V+,Z-axis, Yaxis, X-axis and Ground pins. The ADXL335 runson a low voltage of 3.3V. Arduino Mega was interfaced with the ADXL335 in this experiment. Arduino mega runs on AT Mega microcontroller. Arduino Integrated Design Environment (IDE) is the development environment in which codes were written in C-Language and enabled the microcontroller to synthesize the reading form the accelerometer. The readings of the accelerometer were taken from the serial monitor and this reading correspond to the tilting of the accelerometer in 3-axis, $\mathrm{X}, \mathrm{Y}$ and $\mathrm{Z}$ direction which were in $\mathrm{g}$ acceleration. Accelerometer calculates the acceleration supported with the G-force applied on the axis. Fig.5 shows the connection between the Arduino Mega and ADXL335 using fritzing software while fig. 6 shows the workstation for the experiment which the red circle shows the ADXL335 accelerometer under test.

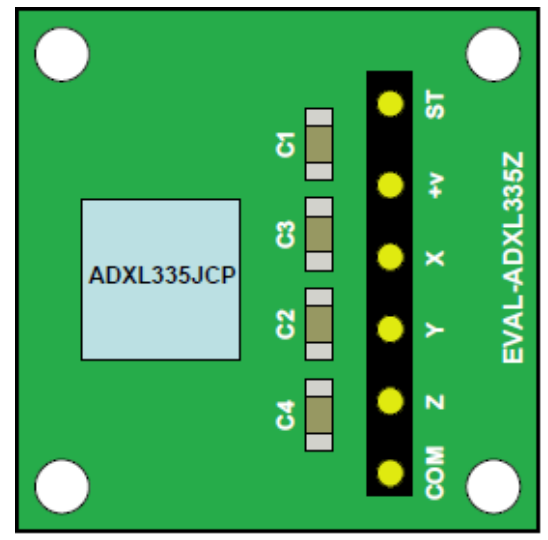

Fig. 4. EVAL-ADXL335Z evaluation board [14]

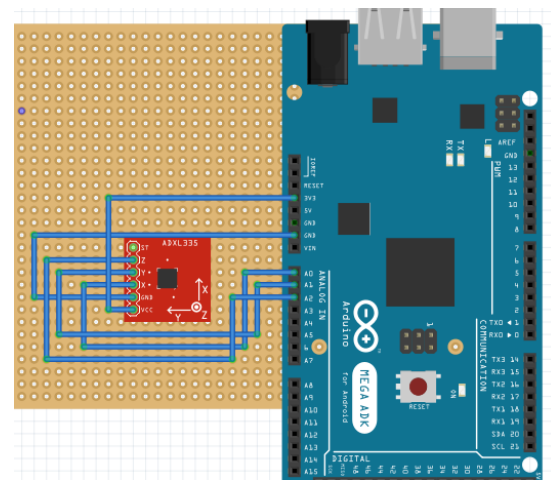

Fig. 5. Connection between the Arduino Mega and ADXL335 


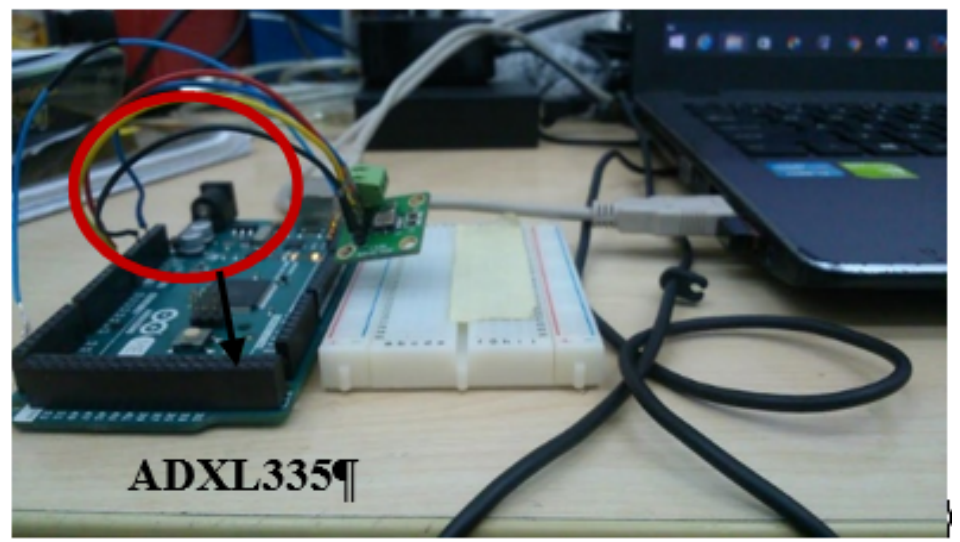

Fig. 6. Practical experiment workstation

\subsection{Optimized performance of accelerometer measurements}

The orientation of the accelerometer can be simplified into rotational matrix. The rotational matrix is a sub-topic of the Euler's angles. The accelerometer gives output in terms of low and high $\pm 1 \mathrm{~g}$ in bottom and up $\mathrm{x}, \mathrm{y}, \mathrm{z}$ side. The other important things to know is that the accelerometer depends on the rotation and movement and gravitational field vector to get its acceleration reading. Therefore, it is necessary to understand key important part of aviation rotational matrices. The pitch $(\varphi)$, roll $(\theta)$ and $\operatorname{yaw}(\psi)$ were the best way to communicate with the accelerometer when there is tilting or movement reading. So, the accelerometer was calibrated first to get the reading then the reading can was filtered and converted to acceleration data for the pitch and roll with the aid of the microcontroller. Anomalies might exist in calculation and this was dealt with proper and well-structured equation to get the pitch and roll to eliminates instabilities. The changes in orientation are described by rotations in roll $\varphi$, pitch $\theta$ and yaw $\psi$ about the $\mathrm{x}, \mathrm{y}$ and $\mathrm{z}$ axes respectively. The accelerometer output is negated to give value $+1 \mathrm{~g}$ in any axis aligned with the earth's downward gravitational field [15]. Consecutively, the 3-axis accelerometer oriented in the earth's gravitational field $g$ and undergoing linear acceleration $a_{r}$ measured in the earth's reference frame $r$, will have output $\mathrm{G}_{\mathrm{p}}$ given by:

$$
G_{p}=\left(\begin{array}{c}
G_{p x} \\
G_{p y} \\
G_{p z}
\end{array}\right)=R\left(g-a_{r}\right)
$$

Where $\mathrm{R}$ is the rotation matrix describing the orientation of accelerometer relative to the earth's coordinate frame.

The Accelerometer has no linear acceleration $\mathrm{a}_{\mathrm{r}} \approx 0$. This assumption is needed to solve (1) for rotation matrix $\mathrm{R}$, and consequences in linear acceleration. This also provides that initial orientation of the accelerometer is lying flat with earth's 
gravitational field aligned with z-axis [15]. Provided that this assumption proves right, the output $\mathrm{G}_{\mathrm{p}}$ is below:

$$
G_{p}=\left(\begin{array}{l}
G_{p x} \\
G_{p y} \\
G_{p z}
\end{array}\right)=R g=R\left(\begin{array}{l}
0 \\
0 \\
1
\end{array}\right)
$$

The roll, pitch and yaw rotation matrices, which transform a vector under a rotation of the coordinate system by angles $\varphi$ in roll, $\theta$ in pitch and $\psi$ in yaw about the $\mathrm{x}, \mathrm{y}$ and $\mathrm{z}$ axes respectively are [15]:

$$
\begin{gathered}
R x(\varphi)=\left(\begin{array}{ccc}
1 & 0 & 0 \\
0 & \cos \varphi & \sin \varphi \\
0 & -\sin \varphi & \cos \varphi
\end{array}\right) \\
R y(\theta)=\left(\begin{array}{ccc}
\cos \theta & 0 & \sin \theta \\
0 & 1 & 0 \\
\sin \theta & 0 & \cos \theta
\end{array}\right) \\
R z(\psi)=\left(\begin{array}{ccc}
\cos \psi & \sin \psi & 0 \\
-\sin \psi & \cos \psi & 0 \\
0 & 0 & 1
\end{array}\right)
\end{gathered}
$$

As discussed earlier, at gravitational field $G_{p}$ measured by acceleration is then apply to the pitch, roll and yaw. In the event of existence of pitch and roll, there are six possible orders of three rotation matrices. Rotation matrix does not mean that composite rotation matrix $\mathrm{R}$ depends on the order in which roll, pitch and yaw rotation are applied [15]. For clarity sake, the rotation matrices $\mathrm{R}$ will be computed to show effect on earth's gravitational field of $1 \mathrm{~g}$ which is shown in equations below.

$$
\begin{gathered}
R x y z\left(\begin{array}{l}
0 \\
0 \\
1
\end{array}\right)=R x(\varphi) R y(\theta) R y(\psi)\left(\begin{array}{l}
0 \\
0 \\
1
\end{array}\right)=\left(\begin{array}{c}
-\sin \theta \\
\cos \theta \sin \varphi \\
\cos \theta \sin \varphi
\end{array}\right) \\
R y x z\left(\begin{array}{l}
0 \\
0 \\
1
\end{array}\right)=R y(\theta) R x(\varphi) R z(\psi)\left(\begin{array}{l}
0 \\
0 \\
1
\end{array}\right)=\left(\begin{array}{c}
-\sin \theta \cos \varphi \\
\sin \varphi \\
\cos \theta \cos \varphi
\end{array}\right) \\
R x z y\left(\begin{array}{l}
0 \\
0 \\
1
\end{array}\right)=R x(\varphi) R z(\psi) R y(\theta)\left(\begin{array}{l}
0 \\
0 \\
1
\end{array}\right)=\left(\begin{array}{c}
\cos \theta \sin \varphi+\cos \varphi \sin \psi \sin \theta \\
\cos \varphi \cos \theta-\sin \theta \sin \varphi \sin \psi
\end{array}\right) \\
R y z x\left(\begin{array}{c}
0 \\
0 \\
1
\end{array}\right)=R y(\theta) R z(\psi) R x(\varphi)\left(\begin{array}{l}
0 \\
0 \\
1
\end{array}\right)=\left(\begin{array}{c}
\cos \theta \sin \varphi \sin \psi-\cos \varphi \sin \theta \\
\cos \psi \sin \theta \\
\cos \theta \cos \varphi+\sin \theta \sin \varphi \sin \psi
\end{array}\right) \\
R z x y\left(\begin{array}{c}
0 \\
0 \\
1
\end{array}\right)=R z(\psi) R x(\varphi) R y(\theta)\left(\begin{array}{c}
0 \\
0 \\
1
\end{array}\right)=\left(\begin{array}{c}
\cos \theta \sin \varphi \sin \psi-\cos \psi \sin \theta \\
\cos \psi \cos \theta \sin \varphi+\sin \theta \sin \psi \\
\cos \theta \cos \varphi
\end{array}\right)
\end{gathered}
$$


$\operatorname{Rzyx}\left(\begin{array}{l}0 \\ 0 \\ 1\end{array}\right)=R z(\psi) R y(\theta) R x(\varphi)\left(\begin{array}{l}0 \\ 0 \\ 1\end{array}\right)=\left(\begin{array}{c}\sin \varphi \sin \psi-\cos \theta \cos \psi \sin \theta \\ \cos \psi \sin \varphi+\cos \varphi \sin \psi \sin \theta \\ \cos \theta \cos \varphi\end{array}\right)$

As seen from (6) to (11) that six composite gives different measurement. Because of this, it is mandatory to define the order in which rotation is to be applied. Starting from (8) to (11), they show complex trigonometric situation, as such they can be cancelled as they involve all the possible orientation and thus cannot be eliminated [15]. (6) and (7) depends only on roll $\varphi$ and pitch $\theta$ angles and are solvable. (6) can be rewritten as:

$$
\frac{G_{p}}{\left\|G_{p}\right\|}=\left(\begin{array}{c}
-\sin \theta \\
\cos \theta \sin \varphi \\
\cos \theta \sin \varphi
\end{array}\right) \Rightarrow \frac{1}{\sqrt{G_{p x^{2}+G_{p y^{2}}{ }^{2} G_{p z}^{2}}^{2}}}\left(\begin{array}{l}
G p x \\
G p y \\
G p z
\end{array}\right)=\left(\begin{array}{c}
-\sin \theta \\
\cos \theta \sin \varphi \\
\cos \theta \sin \varphi
\end{array}\right)
$$

The formula can be further analysed and thus it extends to providing the angles denoted as $\mathrm{x}, \mathrm{y}, \mathrm{z}$ corresponding to the rotation sequence $\mathrm{R} \mathrm{x}, \mathrm{y}, \mathrm{z}$ given below:

$$
\begin{gathered}
\tan \varphi_{x y z}=\left(\frac{G_{p y}}{G_{p z}}\right) \\
\tan \varphi_{x y z}=\left(\frac{-G_{p x}}{G_{p y} \sin \emptyset+G_{p z} \cos \emptyset}\right)=\frac{-G_{p x}}{\sqrt{G_{p y}{ }^{2}+G_{p z}^{2}}}
\end{gathered}
$$

(7) can then can be similarly solved for the roll and pitch angles from an arbitrary accelerometer reading $\mathrm{G}_{\mathrm{p}}$ :

$$
\begin{gathered}
\frac{G_{p}}{\left\|G_{p}\right\|}=\left(\begin{array}{c}
-\sin \theta \cos \varphi \\
\sin \varphi \\
\cos \theta \cos \varphi
\end{array}\right) \Rightarrow \frac{1}{\sqrt{G_{p x^{2}}{ }^{2} G_{p y^{2}}{ }^{2} G_{p z}^{2}}}\left(\begin{array}{l}
G p x \\
G p y \\
G p z
\end{array}\right)=\left(\begin{array}{c}
-\sin \theta \cos \varphi \\
\sin \varphi \\
\cos \theta \cos \varphi
\end{array}\right) \\
\tan \varphi_{y x z}=\frac{-G_{p x}}{\sqrt{G_{p x^{2}+G_{p z}^{2}}^{2}}} \\
\tan \varphi_{y x z}=\left(\frac{-G_{p x}}{G_{p z}}\right)
\end{gathered}
$$

However, limiting roll and pitched angle to lie in between the range of $-180^{\circ}$ to $180^{\circ}$ to reduce the number of possible solution at multiples of $360^{\circ}$ [15]. Then evaluating by adding and subtract angle for pitch angle $\pi^{-} \theta$ and roll angle $\varphi+\pi$.

$$
\left(\begin{array}{c}
-\sin (\pi-\theta) \\
\cos (\pi-\theta) \sin (\varphi+\pi) \\
\cos (\pi-\theta) \sin (\varphi+\pi)
\end{array}\right)=\left(\begin{array}{c}
-\sin \theta \\
\cos \theta \sin \varphi \\
\cos \theta \sin \varphi
\end{array}\right)
$$

The following equation also does the same for the Equation 7 by giving pitch angle an added elevation of $\theta+\pi$ and roll angle $\pi-\varphi$ : 


$$
\left(\begin{array}{c}
-\sin (\theta+\pi) \cos (\pi-\varphi) \\
\sin (\pi-\varphi) \\
\cos (\theta+\pi) \cos (\pi-\varphi)
\end{array}\right)=\left(\begin{array}{c}
-\sin \theta \cos \varphi \\
\sin \varphi \\
\cos \theta \cos \varphi
\end{array}\right)
$$

These two equations did not help much, and thus further solution is to limit either pitch or roll to lie between $-90^{\circ}$ to $90^{\circ}$. In a smartphone, roll angle is $-90^{\circ}$ to $90^{\circ}$ while pitch angle is $-180^{\circ}$ to $180^{\circ}$. The denominator is not supposed to become zero and the equation below shows how to overcome instability [15]. Thus, a way to eliminate the denominator ever being zero is to substitute a fraction $\mu$ (square of accelerometer) $\mathrm{x}$ axis reading $\mathrm{G}_{\mathrm{px}}{ }^{2}$ into denominator. The resulting equation is:

$$
\begin{gathered}
\tan \theta_{x y z}=\frac{-G_{p x}}{\operatorname{sign}\left(G_{p z}\right) \sqrt{G_{p z}{ }^{2}+\mu G_{p y}{ }^{2}}} \\
\tan \theta_{x y z}=\frac{-G_{p y}}{\sqrt{G_{p x}{ }^{2}+G_{p z}{ }^{2}}}
\end{gathered}
$$

To give a better argument, a simple vector algebra mathematically describing a way to calculate the angle change $\alpha$ of apparent gravity vector between two accelerometer readings is given below [15]. This unit are used below which are scalar product $a$. band unit vector $\hat{n}$.

$$
\begin{aligned}
& a \cdot b=\left(\begin{array}{l}
a_{x} \\
a_{y} \\
a_{z}
\end{array}\right) \times\left(\begin{array}{l}
b_{x} \\
b_{y} \\
b_{z}
\end{array}\right)=a_{x} b_{x}+a_{y} b_{y}+a_{z} b_{z}=|a||b| \cos \alpha \\
& \cos \alpha=\frac{a_{x} b_{x}+a_{y} b_{y}+a_{z} b_{z}}{\sqrt{a_{x}^{2}+a_{y}^{2} a_{z}^{2}} \sqrt{b_{x}^{2}+b_{y}^{2} b_{z}^{2}}} \\
& a \times b=\left(\begin{array}{l}
a_{x} \\
a_{y} \\
a_{z}
\end{array}\right) \times\left(\begin{array}{c}
b_{x} \\
b_{y} \\
b_{z}
\end{array}\right)=\left|\begin{array}{ccc}
i & j & k \\
a_{x} & a_{y} & a_{z} \\
b_{x} & b_{y} & b_{z}
\end{array}\right|=\left(\begin{array}{c}
a_{y} b_{z}-a_{z} b_{y} \\
a_{z} b_{x}-a_{x} b_{z} \\
a_{x} b_{y}-a_{y} b_{x}
\end{array}\right)=|a||b| \hat{n} \sin \alpha \\
& \hat{n} \sin \alpha=\left(\frac{1}{\sqrt{a_{x}^{2}+a_{y}^{2} a_{z}^{2}}}\right)\left(\frac{1}{\sqrt{b_{x}^{2}+b_{y}^{2} b_{z}^{2}}}\right)\left(\begin{array}{l}
a_{y} b_{z}-a_{z} b_{y} \\
a_{z} b_{x}-a_{x} b_{z} \\
a_{x} b_{y}-a_{y} b_{x}
\end{array}\right)
\end{aligned}
$$

With a few calculations it could be tested that the reading of the accelerometers was orthogonal to each other [15]. Accelerometer reading is $G_{p}$ in absence of linear acceleration.

$$
G p\left(\begin{array}{l}
0 \\
0 \\
1
\end{array}\right)=G p z=|G p| \cos \rho \Rightarrow \cos \rho=\frac{G p z}{\sqrt{G_{p x^{2}+G_{p y}{ }^{2}+G_{p z}{ }^{2}}}}
$$


After all, the equation depicting the accelerometer pitch and roll has been deduced. This equation describes the calculation of getting the pitch and roll for tilt angle from the accelerometer.

$$
\begin{aligned}
& \operatorname{pitch}=\operatorname{atan}\left(x_{\text {axis }} / \operatorname{sqrt}\left(y_{\text {axis }}{ }^{2}+z_{\text {axis }}{ }^{2}\right)\right. \\
& \operatorname{roll}=\operatorname{atan}\left(y_{\text {axis }} / \operatorname{sqrt}\left(x_{\text {axis }}{ }^{2}+z_{\text {axis }}{ }^{2}\right)\right. \\
& \text { yaw }=\operatorname{atan}\left(z_{\text {axis }} / \operatorname{sqrt}\left(\left(x_{\text {axis }}{ }^{2}+z_{\text {axis }}{ }^{2}\right)\right.\right.
\end{aligned}
$$

By changing into radian for easier programming in Arduino IDE, the formulas become:

$$
\begin{gathered}
\text { Pitch }=\operatorname{pitch}(180 / p i) \\
\text { Roll }=\operatorname{roll}(180 / p i) \\
y a w=\operatorname{yaw}(180 / p i)
\end{gathered}
$$

The most important orientation is getting the voltage output from the accelerometer converted to acceleration and finally getting them into g-acceleration. The total Gforce can be calculated by adding up all the acceleration vectors, which gives the net acceleration.

\subsection{LabView based monitoring system}

National Instruments (NI) LabVIEW software allows virtual instruments (VI) to be built using graphical programming language. As such, LabView Interface for Arduino(LIFA) has been used to make application programmable interface(API) for the experiment. This was done using VI, a software for translating all the Arduino serial com with the LabVIEW software [16]. NI-VISA drivers need to be installed for communication with Arduino board in LabVIEW as Arduino appears as a serial instrument device for LabVIEW. Additionally, the JKI VI Package Manager (VIPM) needs to install as well as it allows to install and update LabVIEW libraries [16]. The LIFA is updated in LabVIEW using VIPM. Arduino IDE is opened so that the LIFA firmware can be upload to the Arduino board. After all the necessary steps are taken, then a simple block is drawn in LabVIEW to get the response from the Accelerometer. Fig. 7 shows the block diagram code in LabView. Fig. 7 which depicts the block diagram consists of the VISA settings for the Arduino input, accelerometer readings for the LIFA and the signal waveform for the accelerometer tilt or movements.NI provides a Data Dashboard for LabVIEW app which is used to create a custom view of NI LabVIEW software applications. The app displays the values of web services on charts for the accelerometer readings. To use the Data Dashboard for LabVIEW, an embedded application must be created that publish the data to the network [17][18]. The Web service used in the setup to create data stream 
from the NI LabVIEW to the android smartphone Data Dashboard was invoked with a remote target which uses standard HTTP protocols. Therefore, the Data Dashboard sends a request to the LabVIEW application, which processes the request and replies with a response. With the LabVIEW Web server, VI's can be deployed as Web services via shared variables using standard IP addresses [19]. After the application is developed and Web services are deployed, building a dashboard inside the app is simple by browsing and choosing the signal to monitor and control. The Data Dashboard used in this experiment was displayed on a smartphone running on android. The Data Dashboard was downloaded from the Google Play Store. This is part of the Internet of Things(IoT) ecosystem which allow data transmission between devices and users.

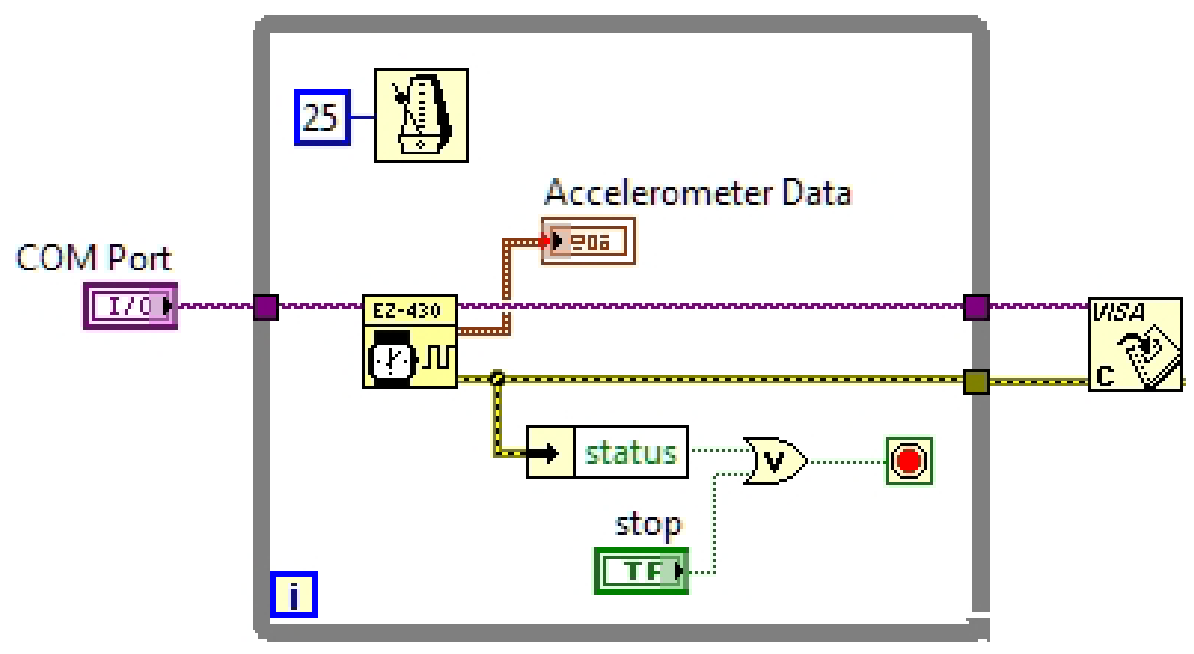

Fig. 7. Block diagram code in LabVIEW

There are steps that needs to be taken before monitoring can be done using the Data Dashboard app. The app is used to create a new Dashboard which can be used to setup an indicator for chart. After that, it is mandatory to deploy shared variables inside LabVIEW by creating a new project that enables VI and this connection must be deployed [19]. In the Data Dashboard app, the Data Link is used to create connection with the shared variable in LabVIEW. The shared variable server DNS or IP address is entered in the shared variables connect dialog. In the case of this project, the IP address is that of the computer being used [19]. This made monitoring possible by pressing the Play button in the Data Dashboard. At this moment, a connection has been established between NI LabVIEW project and the Data Dashboard to enable monitoring service. However, we need to create a web service that allows continuous flow of data with no interruption. The RESTful Web Service enables VI's connection with Data Dashboard which uses HTTP GET and POST. A new lab project is created, and web service option is enabled which created web resources for the new VI and HTTP method. Indicator are created and assigned to connect pane terminal for the VI 
so that Data Dashboard can monitor the output of the web method VI. Thereafter, the Application Web Server is published and deployed. The Dashboard indicators was now linked to the web methods which can be done either with polling and calling. Polling a web method calls the web method continuously every .3 to 30 seconds as specified by the user. Calling a web method only calls the web method when the call button is pressed. In this project, a poling connection was utilized to continuously populate the data on the chart indicator [19]. The poll web service is added to the Dashboard while the web link is chosen. The web service connection will request the user to enter the server DNS or IP address with port of the web service. The web method to link the poll is selected and then the web service is activated successfully. The chart can now be created in Data Dashboard along with web method variables. This made real-time monitoring of the accelerometer possible.

\section{Result and Discussion}

\subsection{Result analysis of PSpice simulation}

PSpice simulation was carried out to see the voltage output against time. Fig. 8 showed the PSpice simulation result. Fig. 8 showcased the curve attained form PSpice simulation which showed that the IEPE interface circuit was responding to the VPWL-source file that was directly input into the simulation. The curve showed that the voltage reading was constantly in $20 \mathrm{mV}$ from time $0 \mu \mathrm{s}$ to $0.6 \mu$ s until it started to display undulating wave-like graph that rises steadily between $0 \mathrm{mV}$ to $-260 \mathrm{mV}$ from time $0.6 \mu \mathrm{s}$ to $1 \mu \mathrm{s}$. This response was recorded according to the supplied source file.

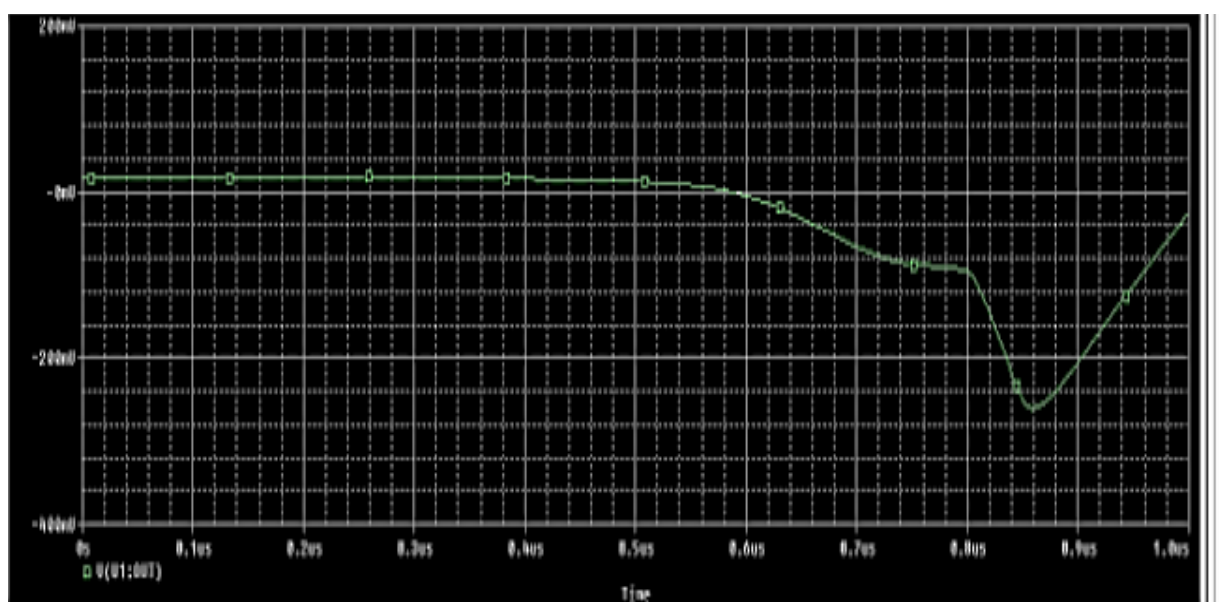

Fig. 8. PSpice simulation result 


\subsection{Result analaysis of laboratory experiment}

The accelerometer response was recorded in the Arduino IDE serial monitor. A tilt was applied on the accelerometer as a representation of micro-movement that might exist in a structure due to a crack or delamination [5]. However, due to limitation of resources and time, the accelerometer was not subject for a test on a structure or material. Notwithstanding, the experiment was a success as the accelerometer response to shake test gave an insight of possible scenarios that could occur when structures or materials goes through delamination. Fig.9 showed part of the recorded data obtained from the serial monitor. In fig. 9, the x-axis showed a changing acceleration as well as the y-axis and z-axis. The readings correspond to the accelerometer movement due to a slight swing on the accelerometer. A slow swing applied to the accelerometer showed a small acceleration while a fast swing applied to the accelerometer showed higher changes in acceleration. Furthermore, the result was further analysed by using least square solution. The linear least square solution is used when the data to be analysed has many equations than variables. The method of least squares is a standard approach in regression analysis to the approximate solution of over determined solutions such as sets of equations in which there are more equations than unknowns. Least squares mean that the overall solution minimizes the sum of the squares of the errors made in the results of every single equation. Least square has two categories which are linear or ordinary least squares and non-linear least squares. The data analysis was done using MATLAB 2015 Edition in this experiment. The non-linear acceleration calculation derived from the microcontroller can be different for each voltage output. The equation for finding the linearity of the matrix for acceleration is:

$$
x=\left(A^{T} A\right)^{-1} A Y
$$

Where $\mathrm{x}$ is the acceleration, $\mathrm{A}$ is the output voltage and $\mathrm{Y}$ is the input voltage. MATLAB served as a very resourceful tool to get all the data in and get the output for each of the acceleration parameters. In the analysis of the result, 26 readings were plotted for the least square graph analysis. The MATLAB function $\operatorname{plot}($ matrix $(A, B))$ was used to generate the graph of least square regression which is displayed in fig.10. In fig. 10, the regression graph showed voltage against acceleration. The acceleration can be seen to be reducing as the voltage decreases while the acceleration increases as the voltage increase. This validate the experiment that when the swing applied to accelerometer is slow the voltage is low, so the acceleration is slow as well. Whereas, when the swing applied to the accelerometer is fast, the voltage is high, and the acceleration is higher as well (see for example [4, 12]. 
Paper-Accelerometer Based Structural Health Monitoring System on the go: Developing Monitoring ..

$$
\mathrm{m} / \mathrm{s}^{2} \quad \mathrm{~m} / \mathrm{s}^{2} \quad \mathrm{~m} / \mathrm{s}^{2} \quad \mathrm{~m} / \mathrm{s}^{2} \quad \mathrm{~m} / \mathrm{s}^{2} \quad \mathrm{~m} / \mathrm{s}^{2}
$$

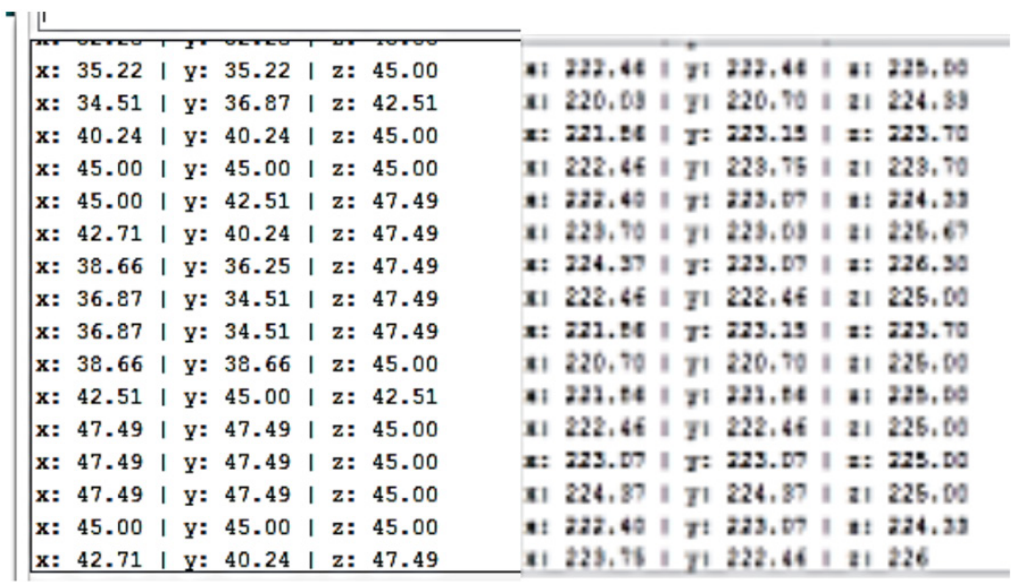

Fig. 9. Part of the data acquired from serial monitor

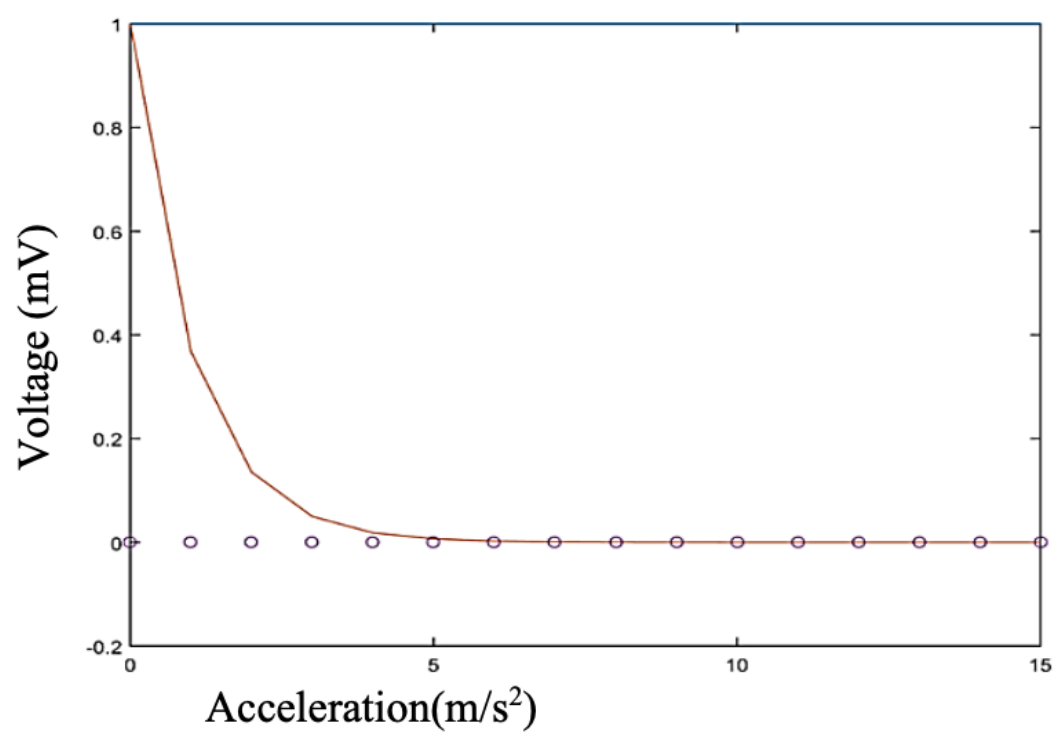

Fig. 10.Least Square regression graph 


\subsection{Reault Analysis of LabVIEW based Monitoring System}

The LabVIEW connection with Arduino was deployed and thus the LabVIEW was able to read the output from the communication port of the computer, which also served the Arduino microcontroller. This establishment made the LabVIEW graphical interface to be used to visualize the vibration of the accelerometer when it was swung. The signal waveform graph from the LabVIEW graphical user interface was in form of Accelerometer signal amplitude against time. The time is continuous and this this gave a real-time visualization of the accelerometer response. Fig. 11 showed the LabVIEW graphical user interface visualization. From Fig. 11, it can be seen that more vibration occurs when the tilt is more towards the negative y-axis which corroborated that the accelerometer can detect micro-movements in form of vibration $[5,13]$. LabVIEW enables information streaming in the graphical interface to be transmitted over the internet to either a smartphone or tablet. In the case of our experiment, a smartphone running android was used to get the real-time monitoring of the data from the accelerometer sensor. The graph indicator was setup in the android smartphone running the Data Dashboard app and the connection to the internet was initialized. Fig. 12 showed the graph indicator for accelerometer axis of $\mathrm{x}, \mathrm{y}$ and $\mathrm{z}$ in NI Data Dashboard. As seen in fig. 12, the Data Dashboard Mobile for LabVIEW app can display only one indicator per dashboard whereas tablets allow multiple indicator to display at once.

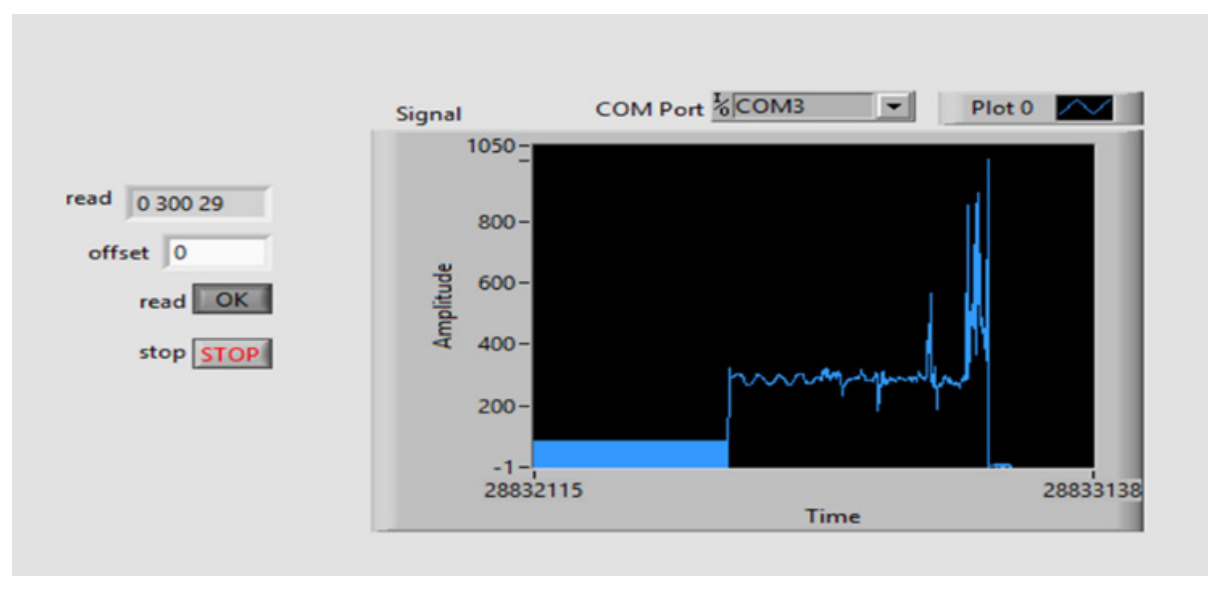

Fig. 11. LabVIEW graphical user interface 


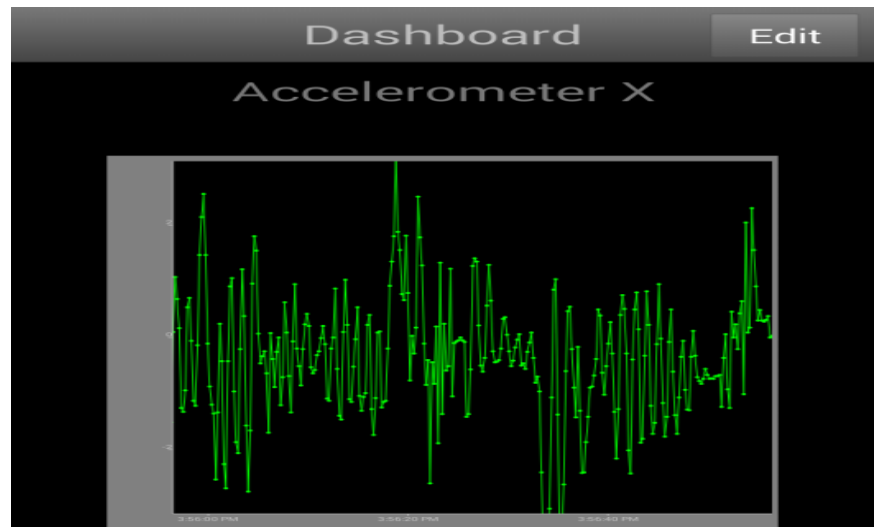

a)

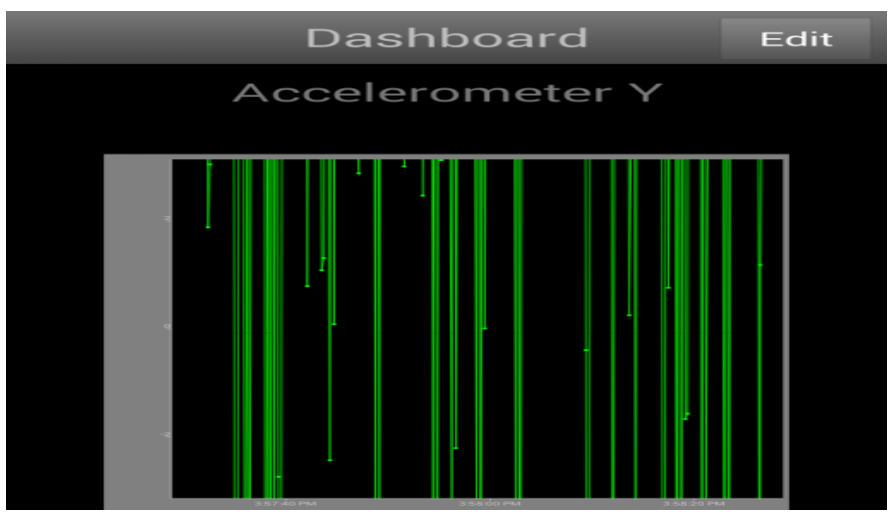

b)

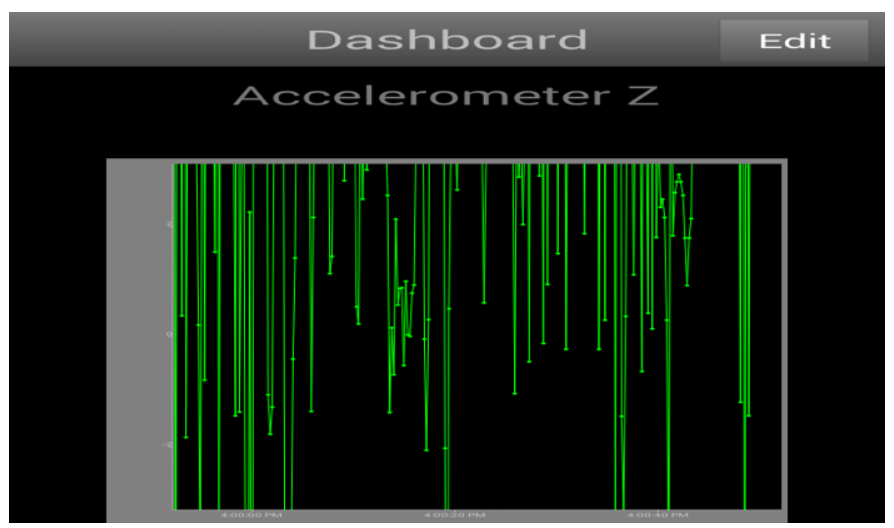

c)

Fig. 12.a): Data Dashboard accelerometer display of $x$-axis, b): Data Dashboard accelerometer display of y-axis and c): Data Dashboard accelerometer display of z-axis 


\section{Conclusion}

SHM is a very crucial assessment of part of development of infrastructure whether buildings or bridges. Previous work on SHM have not primarily dealt with transmission of information attained from SHM systems, which is an essential part of early warning system to reduce avoidable disaster. As information dissemination serves as a critical part of SHM system, hence the simulation and experiment carried out in this study has shown that vibrational analysis of structures can be very advantageous when it is equipped in the planning of a project. SHM can help in maintenance of building and structures as well as making the process of management of structures automated. The PSpice simulation showed the effect of verifying the theory before setting up practical experiment. The performance of ADXL335 accelerometer was tested in this investigation and the result attained showed that vibration measurement with the use of accelerometer is effective for SHM. Furthermore, LabVIEW software was used as an embedded software via Arduino IDE to output accelerometer readings through graphical chart inside LabVIEW graphical user interface. A web service that enabled real-time monitoring of the accelerometer was also deployed which enabled smartphone to be able to receive data from the LabVIEW graphical user interface. This is an Internet of Things (IoT) application enabling SHM to be easily monitored and controlled anywhere the user might be. However, several other factors are still required to ascertain the capability of this experiment in real-life situation. Future work might involve placing the accelerometer in a structure or material to test its performance.

\section{Acknowledgement}

This work is partially supported by International Islamic University Malaysia, Research Initiative Grant Scheme no. RIGS17-023-0598. It was conducted at IoT \& Wireless Communication Protocols Lab, ECE/KoE/IIUM.

\section{References}

[1] Mutlib, N. K., Baharom, S. B., El-Shafie, A., \& Nuawi, M. Z. (2016). Ultrasonic health monitoring in structural engineering: buildings and bridges. Structural Control and Health Monitoring, 23(3), 409-422. https://doi.org/10.1002/stc.1800

[2] Alam, A. Z., Hassan, N. S. A. A., Mustapha, N. A. C., Islam, M. R., Khan, S., \& Eusuf, M. A. (2016). Wireless Capacitor Sensing for Structural Health Monitor. In Proceedings of the World Congress on Engineering (Vol. 1).

[3] Chanv, B., Bakhru, S., \& Mehta, V. (2017, December). Structural health monitoring system using IOT and wireless technologies. In 2017 International Conference on Intelligent Communication and Computational Techniques (ICCT) (pp. 151-157). IEEE. https://doi.org/10.1109/INTELCCT.2017.8324036 
[4] Bedon, C., Bergamo, E., Izzi, M., \& Noè, S. (2018). Prototyping and Validation of MEMS Accelerometers for Structural Health Monitoring-The Case Study of the Pietratagliata Cable-Stayed Bridge.

[5] Amezquita-Sanchez, J. P., \& Adeli, H. (2016). Signal processing techniques for vibrationbased health monitoring of smart structures. Archives of Computational Methods in Engineering, 23(1), 1-15. https://doi.org/10.1007/s11831-014-9135-7

[6] Valinejadshoubi, M., Bagchi, A., \& Moselhi, O. (2016). Structural Health Monitoring of Buildings and Infrastructure. Structural Health Monitoring, 1, 50371.

[7] Mustapha, N. A. C., Alam, A. Z., Khan, S., \& Azman, A. W. (2016). Efficient capacitance sensing for wireless health monitoring system. IIUM Engineering Journal, 17(2), 21-29. https://doi.org/10.31436/iiumej.v17i2.609

[8] Al-Adnani, N. N. A., Mustapha, F., Sapuan, S. M., \& Saifulnaz, M. R. (2016). Structural health monitoring and damage identification for composite panels using smart sensor. Journal of Intelligent Material Systems and Structures, 27(17), 2313-2323. https://doi.org/10.1177/1045389X16629553

[9] Kinet, D., Mégret, P., Goossen, K. W., Qiu, L., Heider, D., \& Caucheteur, C. (2014). Fiber Bragg grating sensors toward structural health monitoring in composite materials: Challenges and solutions. Sensors, 14(4), 7394-7419. https://doi.org/10.3390/s140407394

[10] Feng, D., \& Feng, M. Q. (2017). Experimental validation of cost-effective vision-based structural health monitoring. Mechanical Systems and Signal Processing, 88, 199-211. https://doi.org/10.1016/j.ymssp.2016.11.021

[11] Behnia, A., Chai, H. K., \& Shiotani, T. (2014). Advanced structural health monitoring of concrete structures with the aid of acoustic emission. Construction and Building Materials, 65, 282-302. https://doi.org/10.1016/j.conbuildmat.2014.04.103

[12] Bhuiyan, M. Y., Bao, J., Poddar, B., \& Giurgiutiu, V. (2018). Toward identifying cracklength-related resonances in acoustic emission waveforms for structural health monitoring applications. Structural Health Monitoring, 17(3), 577-585. https://doi.org/10.1177/1475921717707356

[13] Janapati, V., Kopsaftopoulos, F., Li, F., Lee, S. J., \& Chang, F. K. (2016). Damage detection sensitivity characterization of acousto-ultrasound-based structural health monitoring techniques. Structural Health Monitoring, 15(2), 143-161. https://doi.org/10.1 $177 / 1475921715627490$

[14] ADXL335. (n.d). Retrieved from

[15] http://www.analog.com/en/products/adxl335.html\#product-samplebuy. Retrieved on 22 August 2018.

[16] Pedley, M. (2013). Tilt sensing using a three-axis accelerometer. Freescale semiconductor application note, 1, 2012-2013. Retrieved on 23 August 2018

[17] Sammy K.(2011). LABVIEW INTERFACE FOR ARDUINO DOCUMENTS. Rettrieved from https://forums.ni.com/t5/LabVIEW-Interface-for-Arduino/LabVIEW-Interface-forArduino-Setup-Procedure/ta-p/3521346. R3521346. n 20 August 2018

[18] Smartphones and Tablets for Measurement and Control. (2014). Retrieved from http://www.ni.com/newsletter/51387/en/ . Retrieved on 21 August 2018

[19] S.V. Manikanthan, T.Padmapriya, "United Approach in Authorized and Unauthorized Groups in LTE-A Pro", Jour of Adv Research in Dynamical \& Control Systems, Vol. 10, 10-Special Issue, 2018, pp. (1137-1145).

[20] Getting Started with Data Dashboard for LabVIEW. (2014). Retried from http://www.ni.com/tutorial/13757/en/. Retrieved on 20 August 2018 


\section{Authors}

Salami Ifedapo Abdullahi is with the department of Electrical and Computer Engineering, Kulliyyhah of Egnieering, International Islamic University Malaysia (IIUM) - Gombak, 53100 KL Malaysia.

Nurul Arfah Che Mustapha is with the department of Electrical and Computer Engineering, Kulliyyhah of Egnieering, International Islamic University Malaysia (IIUM) - Gombak, 53100 KL Malaysia.

Mohamed Hadi Habaebi is with the department of Electrical and Computer Engineering, Kulliyyhah of Egnieering, International Islamic University Malaysia (IIUM) - Gombak, 53100 KL Malaysia.

Md. Rafiqul Islam is with the department of Electrical and Computer Engineering, Kulliyyhah of Egnieering, International Islamic University Malaysia (IIUM) - Gombak, 53100 KL Malaysia.

Article submitted 2019-02-08. Resubmitted 2019-03-15. Final acceptance 2019-03-17. Final version published as submitted by the authors. 